\title{
O MODELO DO SISTEMA DE ORÇAMENTO CORPORATIVO: INFLUÊNCIAS NO COMPORTAMENTO E NO DESEMPENHO GERENCIAL ${ }^{1}$
}

\section{THE DESIGN OF CORPORATE BUDGETING SYSTEMS: INFLUENCES ON MANAGERIAL BEHAVIOR AND PERFORMANCE}

Kenneth A. MERCHANT

Professor assistente de Gestão de Negócios na Universidade de Harvard

E-mail: kmerchant@marshall.usc.edu

\begin{abstract}
Resumo
Este trabalho estuda como as diferenças dos sistemas de orçamento, em nível corporativo, estão relacionadas ao tamanho, à diversificação e ao grau de descentralização da corporação, e como as diferentes escolhas de modelo e uso do sistema estão relacionadas ao desempenho organizacional, à motivação e às atitudes gerenciais. Foi desenvolvido um modelo a partir de resultados relatados em pesquisas sobre contabilidade e comportamento organizacional. As expectativas nesse modelo foram exploradas com dados colhidos em 19 indústrias do setor de eletrônicos. Os resultados, geralmente, validam o modelo. Eles mostram que o orçamento, como parte da estratégia de controle da corporação, é relacionado ao contexto corporativo. Empresas maiores tendem a fazer um uso relativamente maior de administrações mais formais, em oposição aos controles interpessoais. Em todas as empresas, os processos mais formais e elaborados de orçamento são, freqüentemente, bem recebidos pelos gerentes, mas nas empresas maiores eles parecem ser mais positivamente relacionados ao desempenho.
\end{abstract}

\begin{abstract}
This study investigates how differences in corporate-level budgeting systems are related to corporate size, diversity, and degree of decentralization, and how different choices in system design and use are related to organizational performance and manager motivation and attitudes. A model was developed from related findings in research in accounting and organizational behavior, and the expectations in the model were explored with data gathered from 19 firms in the electronics industry. The results generally support the model. They show that budgeting, as part of the corporate control strategy, is related to the corporate context. Larger firms tend to make relatively high use of more formal administrative, as opposed to interpersonal, controls. In all firms, the more formal and elaborate budgeting processes are generally received well by the managers, but in larger firms they appear to be more positively linked with performance.
\end{abstract}

Traduzido em 31/11/2007 • Publicado em 31/12/2007

\footnotetext{
${ }^{1}$ Essa pesquisa foi financiada, em parte, por doações da Peat, Marwick, Maitchel \& Co. Fundação e Programa Profissional de Contabilidade, Universidade da Califórnia, Berkley. 0 autor agradece pelas valiosas sugestões de John T. Wheeler, Peter Brownel e de revisores anônimos.

N.T. Revisão Técnica de Emerson Tadeu G. Rici. Tradução de Priscilla Ferro Rici, do original em inglês The Design of Corporate Budgeting Systems: Influences on Managerial Behavior and Performance, in The Accounting Review, LVI, p. 813829. A American Accounting Association $\odot$ não revisou esta tradução e não pode, portanto, ser responsabilizada por eventuais falhas.
} 


\section{INTRODUÇÃO}

Um sistema de orçamento é uma combinação de fluxo de informação, processos e procedimentos administrativos que, geralmente, é parte integral do planejamento de curto prazo e do sistema de controle de uma organização. Pesquisadores têm notado a importância do orçamento e têm conduzido pesquisas relacionadas ao tema, mas os resultados têm sido de difícil integração e, freqüentemente, conflitantes.

A principal razão para os resultados confusos pode ser o fato de que a maior parte dos estudos empíricos, provavelmente por razões de facilitação metodológica, enfoca as análises de níveis mais baixos, geralmente, em indivíduos. Um tipo comum de estudo examina os efeitos de características do orçamento, tais como a participação (Milani, 1975; Seafross, 1976), dificuldade em alcançar os resultados (Stedry e Kay, 1966; Kennis, 1979) ou pressão no orçamento (DeCoaster e Fertakis, 1968) no comportamento e nas atitudes gerenciais. 0 outro tipo trata de características orçamentárias como variáveis dependentes e enfoca variáveis independentes tais como a personalidade do gerente (Foran e DeCoster, 1974; Swieringa e Moncor, 1975) ou o tipo de liderança do superior imediato (Hopwood, 1972, 1974). A maioria dos estudos considerou apenas uma pequena quantidade de situações. Essa limitação tem levado a resultados conflitantes, pois o comportamento orçamentário e seus resultados são mais propensos a interagir com características de nível mais alto tal como o tipo de organização ou cultura (Moberg e Koch, 1975; Camman 1976; Otley, 1978; Hopwood, 1978; Shepard e Hougland, 1978; Mealiea e Lee, 1979).

Reconheceu-se a necessidade de integrar resultados e desenvolver estruturas para futuras pesquisas. Um número de estruturas de contingência tem sido publicado na literatura sobre contabilidade (Sathe, 1975; Gordon e Miller, 1976; Ansari, 1977, 1979; Gordon et al.; 1978; Waterhouse e Tiessen, 1978; Banbury e Nahapiet, 1979). Esses artigos são desenvolvidos, principalmente, a partir de dados sobre comportamento organizacional e salientam a importância das características da organização e de seu ambiente de trabalho (por exemplo, a incerteza ambiental, o tamanho da empresa, a sofisticação tecnológica) no modelo da operação dos sistemas de informação da contabilidade, como o orçamento. Enfatiza-se também que tem havido muito poucas pesquisas relacionadas à contabilidade, especialmente pesquisas empíricas, conduzidas em níveis de análise mais altos (ou seja, acima do nível individual). 0 objetivo deste artigo é relatar os resultados de um estudo empírico desenvolvido, visando essa necessidade específica.

A estrutura básica do estudo é apresentada na Figura 1. 0 estudo tem três objetivos principais. Em primeiro lugar, o orçamento é considerado em um nível amplo do sistema e enfoca algumas variáveis do modelo do sistema de orçamento de nível corporativo. Essas variáveis foram anteriormente estudadas apenas conceitualmente, em respeito aos detalhes de informações e à freqüência de planos de atualização, relacionando-as à descrição do contexto corporativo e do comportamento do orçamento. Em segundo lugar, ele tenta explorar a noção da teoria da contingência de que o desempenho organizacional é maior onde há um "casamento" entre o conteúdo corporativo e o modelo e a operação do sistema orçamentário. Por fim, ele repete, em parte, um estudo de orçamento de nível corporativo feito por Bruns e Waterhouse (1975). 
Figura I - Modelo para o estudo

\begin{tabular}{|l|}
\hline CONTEXTO \\
CORPORATIVO \\
- Tamanho \\
- Diversificação \\
- Nível de \\
descentralização \\
\\
\end{tabular}

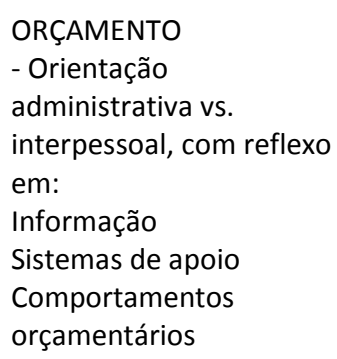

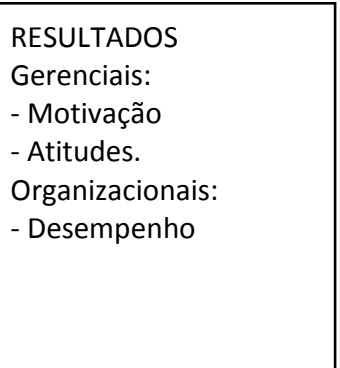

\section{Conteúdo Corporativo e Orçamento}

Normalmente, aceita-se que o orçamento é usado de maneiras diferentes em empresas diferentes. Algumas das variações nas abordagens podem ser explicadas pelo exame das influências externas à organização, como a incerteza no ambiente de trabalho (Burns e Stalker, 1961; Gordon e Miller, 1976; Henderson e Nutt, 1978). No entanto, uma porção significativa da variação também pode ser explicada pelo exame dos aspectos do contexto corporativo, um termo que geralmente se refere ao ambiente de trabalho interno da corporação, no qual o orçamento deve ser operado. Este estudo enfoca os três aspectos relacionados ao contexto corporativo o tamanho da organização, sua diversificação e seu nível de descentralização - que parecem ser fortemente relacionados às escolhas das estratégias de controle organizacional e, consequentemente, orçamentárias.

0 aumento do tamanho das organizações e sua diversidade criam problemas no controle social, na comunicação e na coordenação. Organizações pequenas e que atuam em apenas um tipo de negócio podem ser controladas por mecanismos de controle amplamente mais informais e pessoalmente orientados, tal como a supervisão direta e as comunicações orais. Organizações maiores, no entanto, encontram um número exponencialmente crescente de canais que requerem fluxo de informações para objetivos de coordenação e organizações mais diversificadas enfrentam maiores diferenças nas “orientações cognitivas e emocionais entre os gestores" (Lawrence e Lorsch, 1967, p.11) o que torna as comunicações mais difíceis. Como uma conseqüência, conforme as organizações crescem e se tornam mais diversificadas, elas tendem a descentralizar e implementar uma estratégia de controle mais administrativamente orientada que envolve maior estruturação de atividades, maior formalização nas comunicações e um aumento no uso de informações padronizadas para avaliação do desempenho gerencial (Bruns e Waterhouse, 1975). Controles voltados à administração são discutidos na literatura com muitos títulos diferentes, incluindo "mecanismo impessoal" (Blau e Scott, 1962), “controles formais" (Child, 1973, Woodward, 1970), e "controles de produção" (Ouchi, 1977; Ouchi e Maguire, 1975).

Uma estratégia de controle administrativo que é mais provável em empresas maiores, mais diversificadas e mais descentralizadas, deveria ser consistente com os seguintes sistemas de orçamento e características de comportamento orçamentário:

1) Maior participação da gerência média e baixa nas atividades relacionadas ao orçamento. A participação em níveis inferiores é consistente com o significado de descentralização e Bruns e Waterhouse (1975) afirmam que a participação no orçamento estava relacionada à participação em outras decisões. Isso não surpreende, pois, em empresas mais diversificadas, gerentes de níveis inferiores são, provavelmente, melhor informados sobre as capacidades de suas atividades especializadas e, envolvê-los no 
orçamento tende a render planejamentos mais realistas e fornecer efeitos de motivação positivos.

2) Mais importância dada ao alcance dos planos orçamentários. Um uso do orçamento é informar as unidades organizacionais das intenções das outras unidades e da corporação. Se os objetivos do orçamento são tratados como um compromisso organizacional, o número de exceções ao plano é menor e faz-se necessária menos comunicação para manter a coordenação (Glabraith, 1977).

3) Padrões mais formais de comunicação. Em organizações menores e mais homogêneas, muitas comunicações das intenções da organização e da avaliação gerencial podem ser conseguidas informalmente, por meio de interação interpessoal direta. Em organizações maiores, todavia, muito dessa comunicação é conseguido por meio de canais mais formalizados, incluindo um plano e um sistema orçamentários bem desenvolvidos (Bruns e Waterhouse, 1975; Gordon e Miller, 1976).

4) Maior sofisticação no sistema orçamentário. Inclui maior uso de computadores, pessoal qualificado e modelagem financeira. Por conta da indivisibilidade dos recursos e das economias de escala e maior especialização técnica possível nos componentes administrativos, grandes organizações têm possibilidade de usar recursos mais sofisticados, como planejamento formal e orçamento para os problemas de controle mais complexos (Gordon e Miller, 1976; Banbury e Nahapiet, 1979).

Por outro lado, o orçamento em empresas que usam a estratégia de controle interpessoal, muito provavelmente em empresas menores, menos diversificadas e mais centralizadas, deve ter características opostas.

\section{Efeito na Motivação e nas atitudes}

A maior evidência relacionada ao orçamento na motivação e nas atitudes sugere que essas cognições tendem a ser mais favoráveis em organizações que usam o orçamento como parte da estratégia de controle administrativo. A participação, provavelmente o aspecto mais estudado do orçamento, tem se mostrado positivamente correlacionada com a motivação e/ou o desempenho (Vroom, 1964; Hofstede, 1967; Milani, 1975; Steers, 1976 e Kennis, 1979). Enquanto as evidências sugerem que as relações positivas podem ser generalizadas, deve-se tomar cuidado pois os efeitos da interação de algumas influências culturais (French et. al., 1960) e os traços de personalidade (Brownwll, 1981) têm mostrado diferenças nos sinais de relacionamento. Num estudo de nível organizacional dos Estados Unidos, no entanto, o resultado mais provável é uma relação positiva entre o uso administrativo do orçamento e a motivação e atitude.

\section{Efeito no desempenho}

Enquanto o orçamento pode ser diretamente ligado à motivação e às atitudes, suas relações com o desempenho parecem ser mais complexas. Muitos autores têm tentado encontrar relações simples de duas variáveis, entre os usos do orçamento e o desempenho, sem bons resultados. Kenis (1979) encontrou poucas ligações positivas (mas estatisticamente significativas) entre a ênfase no orçamento e a participação, e ambos, o orçamento e o desempenho na profissão, mas a maior parte dos estudiosos, incluindo Milani (1975), Steers (1976) e Ivancevich (1976), não encontrou tal relação.

Alguns problemas empíricos são relacionados ao método, uma vez que os efeitos do desempenho são difíceis de ser isolados em campo, mas uma explicação mais significativa para a falta de resultados úteis está, provavelmente, relacionada à teoria. As teorias são demasiado simples e os estudos empíricos não têm medido ou controlado as influências interativas significativas nas relações entre o orçamento e o desempenho. É o que afirma 
Hopwood (1976, p. 79) a respeito de uma variável relacionada ao orçamento: a participação.

Enquanto parece que um aumento na participação nas tomadas de decisão pode aumentar a disposição, seu efeito na produtividade é, na melhor das hipóteses, duvidoso, aumentando sob certas circunstâncias, mas possivelmente, até diminuindo sob outras circunstâncias. O problema prático está na tentativa de identificar quais fatores condicionais determinam o maior impacto de um tipo particular de um programa de gerência participativa.

A identificação dos fatores condicionais mencionados por Hopwood é a abordagem da contingência para o modelo organizacional que, segundo Child (1977), tem se tornado um paradigma dominante na teoria organizacional. A noção básica, expressa tanto na literatura de comportamento organizacional (por exemplo, em Kast e Roseinzweig, 1973; Luthans e Stewart, 1977) quanto na anteriormente mencionada estrutura da contabilidade, é que, para o máximo efeito, a modelagem e o uso dos sistemas administrativos, como o orçamento, devem variar de acordo com o cenário. Em termos das variáveis de contexto corporativo examinadas nesse estudo - tamanho, diversificação e dimensão da descentralização - as proposições "normativas" contidas na estrutura de contingência relacionada ao orçamento discutidas acima, sugerem que o desempenho deve ser mais alto nas empresas maiores, mais diversificadas e mais descentralizadas, que tendem a adotar uma estratégia de controle administrativo. As empresas menores, menos diversificadas e mais centralizadas tendem a adotar uma estratégia de controle interpessoal. Embora essa hipótese de "casamento" possa ser afirmada, o apoio empírico a ela é muito limitado neste ponto.

\section{HIPÓTESE}

A discussão anterior pode ser resumida com as seguintes hipóteses:

1. Empresas maiores, mais diversificadas e mais descentralizadas tendem a usar uma estratégia de controle administrativo que, em termos de sistema e comportamento orçamentários, é consistente com:

a. maior participação de gerentes que atuam em níveis hierárquicos médios e baixos nas atividades relacionadas ao orçamento;

b. maior importância no alcance dos objetivos dos planos de orçamento;

c. padrões mais formais de comunicação, e

d. maior sofisticação dos sistemas orçamentários.

2. As motivações e as atitudes dos gestores de nível médio em relação ao orçamento são maiores em empresas que usam o sistema de orçamento administrativamente orientado.

3. O desempenho organizacional é maior quando há um "casamento" entre a estratégia de controle e o contexto corporativo, como descrito na Hipótese 1. 


\section{MÉTODO}

\section{Amostra}

Dezenove indústrias do setor de eletrônicos concordaram em participar do estudo. Uma única indústria foi escolhida para aumentar a receptividade das empresas em relação à participação no estudo e para servir de controle das variáveis que não estavam sendo medidas. A indústria eletrônica foi selecionada porque as empresas desse ramo são diversificadas em tamanho e em orientação administrativa.

A pesquisa teve início pela entrevista com o funcionário de cargo mais alto da companhia, como, por exemplo, o vice-presidente financeiro, o controller, ou o diretor de planejamento e de orçamento. Essa entrevista foi usada para obter informações sobre a empresa, sobre seu planejamento corporativo e sistemas orçamentários e para identificar uma amostra de gerentes de produção de nível médio que estavam ativamente envolvidos no orçamento.

Os gerentes foram selecionados pelo funcionário entrevistado, pois as empresas foram relutantes ou incapazes de fornecer listas completas para uma seleção mais aleatória. 0 número de gerentes selecionados variou de 3 a 21 , dependendo do tamanho da organização e da disposição de ter seus gerentes "perdendo tempo" com o estudo.

Questionários, considerando o comportamento e as atitudes orçamentárias, foram enviados a cada um dos 201 gerentes selecionados em seus ambientes de trabalho. Solicitou-se aos entrevistados que enviassem aos gerentes um memorando alertando-os de que a empresa havia autorizado o estudo. 0 pedido inicial produziu 135 respostas e o acompanhamento rendeu mais 35 , num total de 170 respostas úteis (85\%). Na média, os respondentes tinham 41 anos de idade, já trabalhavam para seus empregadores há 11 anos e mantinham suas posições há quatro. Uma vez que esse foi um estudo de nível corporativo, foi feita uma média das respostas dos gerentes das mesmas empresas e o resultado foi usado como um indicador das atitudes e dos comportamentos orçamentários gerais da empresa.

\section{Medidas}

Características das corporações. As medidas de três características organizacionais foram coletadas das entrevistas:

1. O tamanho foi medido pelo número de empregados.

2. A melhor medida de diversificação é provavelmente aquela desenvolvida por Rumelt (1974) que organiza as empresas em quatro amplas categorias - negócio único (i.e., não diversificada), negócio dominante, negócio relacionado e negócio não-relacionado (i.e., diversificada). Contudo, essa classificação não fornece muita discriminação entre empresas na indústria de eletrônicos, pois muitas são estreitamente focadas em negócios de alta tecnologia. Com isso, a proporção da receita resultante especificamente dos negócios relacionados à eletrônica, multiplicados por menos um $(-1)$, foi usada como um indicador rigoroso da diversificação dessas empresas na indústria eletrônica.

O grau de descentralização e extensão de tomada de decisões em níveis mais baixos da hierarquia corporativa foi medido pela adaptação de um instrumento desenvolvido pelo grupo Aston (por exemplo, Pugh et. al., 1969; Inkson et. al., 1970). É dada uma pontuação para o nível de organização no qual cada uma das 23 decisões-padrão pode ser feita (por exemplo, demitir um supervisor). O alcance teórico dessa medição é de 0 a 115, com uma baixa pontuação evidenciando descentralização e vice-versa. 
Orçamento. A maior parte das empresas tem rotinas de planejamentos múltiplos, com horizontes de planejamento e níveis de detalhes diferentes. Porque os sistemas variam muito e a medição nessa área não é bem desenvolvida, apenas indicadores brutos do modelo de sistema puderam ser usados. As informações a seguir, o apoio do sistema e as variáveis do comportamento orçamentário, foram selecionados como úteis para a distinção entre usos administrativo e interpessoal orçamentários. A informação e os dados de apoio do sistema foram obtidos pelos entrevistadores corporativos, e os dados comportamentais, conseguidos com os gerentes de produção.

\section{A. Informação}

1. A frequiência de atualização dos planos anuais foi medida em uma escala de 1 (anualmente) a 4 (mensalmente ou mais frequentemente).

2. A indicação do detalhamento de informação no planejamento anual foi obtida pela medição da menor fração de tempo (por exemplo, um mês, um trimestre) para o qual os dados foram preparados. A escala usada abrangeu de 1 (períodos maiores que um trimestre) a 4 (um mês ou menos).

Essas medidas tornam operacionais as características de informação identificadas por Gordon et. al. (1978) como potencialmente importantes para o modelo de sistema de informação.

\section{B. Sistemas de apoio}

1. A sofisticação do sistema de apoio computacional para o sistema orçamentário foi medida numa escala de 1 (sem apoio computacional) a 4 (disponibilidade de terminais remotos num modo interativo).

2. 0 uso de um manual de planejamento (sim/não) foi usado como um indicador de apoio à equipe e formalidade do sistema.

3. 0 uso de orçamento de base zero ( $\operatorname{sim} /$ não) foi usado como um indicador da formalidade do sistema e sua sofisticação, uma vez que o ZBB ${ }^{2}$ é uma das estruturas de orçamento mais bem elaboradas.

4. A ligação do sistema de orçamento com o sistema de remuneração da corporação percebida pelos gerentes de nível médio, foi medida pelo uso de uma escala de sete itens sugerida por Hackman e Porter (1975) e usada por Dermer (1975). Um item da amostra dessa escala é: “o desempenho do orçamento é um fator importante no avanço da minha carreira". A média interna de consistência de confiabilidade dessa escala, como medida pelo Alfa de Cronbach, foi 0,79, seguramente acima dos menores limites de aceitabilidade, geralmente considerados entre 0,50 e 0,60 (Nunnally, 1967).

\section{Comportamentos relacionados ao orçamento}

Esse instrumento desenvolvido por Swieringa e Moncur (1975), com modificações insignificantes, foi usado para medir os comportamentos relacionados ao orçamento dos gerentes de produção. 0 orçamento foi definido como plano para períodos maiores ou iguais a um ano. Pediu-se aos gerentes que indicassem a freqüência, na escala de 1 (nunca) a 5 (sempre), onde cada uma das 42 atividades relacionadas ao orçamento se encaixava em seu próprio orçamento.

Esses dados foram fatores de análise para descobrir padrões ocultos e reduzir a dimensionalidade dos itens, ou seja, onde as pontuações de itens múltiplos eram altamente correlacionadas umas com as outras, eram criadas variáveis compostas, capturando a maior parte da variância total. Isso simplifica a análise, pois reduz drasticamente o número de variáveis a ser considerado.

\footnotetext{
${ }^{2}$ N.T. Designação inglesa para traduzir o orçamento construído sem aplicação direta de coeficientes sobre valores históricos.
} 
O fator de análise foi conduzido segundo o método de fatores induzidos do SPSS (Pacote Estatístico para Ciências Sociais) (Nie et. al., 1975). Seis fatores com autovalor maior do que 1,0 foram extraídos, totalizando $81,4 \%$ da variância. A tabela 1 mostra a sobrecarga do fator depois de uma rotação oblíqua Oblimin direta (com $\sigma=0)$, os itens de sobrecarga > 0,40 em cada fator, e a porcentagem da variância explicada em cada fator.

Tabela 1 - Sobrecarga de fatores de itens comportamentais relacionados ao orçamento

\begin{tabular}{|c|c|c|c|}
\hline Fator & Título e sobrecarga de itens $>0,40$ & Sobrecarga & $\begin{array}{l}\text { Porcentagem de } \\
\text { variância }\end{array}$ \\
\hline 1 & $\begin{array}{l}\text { EXPLICAÇÕES DE VARIÂNCIA NECESSÁRIAS } \\
\text { Eu preciso entregar uma explicação por escrito sobre } \\
\text { causas de grandes variâncias de orçamento. } \\
\text { Eu preciso relatar ações que tomo para corrigir causas } \\
\text { de variância de orçamento. } \\
\text { Eu preciso preparar relatórios comparando resultados } \\
\text { reais com o orçamento. } \\
\text { Eu preciso rastrear a causa das variâncias de } \\
\text { orçamento para grupos ou para indivíduos dentro do } \\
\text { meu departamento. }\end{array}$ & $\begin{array}{l}0,77 \\
0,73 \\
0,66 \\
0,66\end{array}$ & 42,6 \\
\hline 2 & $\begin{array}{l}\text { INFLUÊNCIA NOS PLANOS DE ORÇAMENTO } \\
\text { O orçamento está finalizado somente quando eu estou } \\
\text { satisfeito com ele. } \\
\text { Novos orçamentos incluem as mudanças que eu sugeri. }\end{array}$ & $\begin{array}{l}0,67 \\
0,66\end{array}$ & 13,4 \\
\hline 3 & $\begin{array}{l}\text { INTERAÇÕ̃ES COM SUBORDINADOS } \\
\text { Eu discuto os itens do orçamento com meus } \\
\text { subordinados quando ocorrem problemas. } \\
\text { Eu trabalho com meus subordinados na preparação do } \\
\text { orçamento para meu departamento. } \\
\text { Eu avalio meus subordinados por meio dos orçamentos. }\end{array}$ & $\begin{array}{l}0,67 \\
0,57 \\
0,53\end{array}$ & 8,8 \\
\hline 4 & $\begin{array}{l}\text { REAÇÕES AO ENDIVIDAMENTO PREVISTO PELO } \\
\text { ORÇAMENTO } \\
\text { Eu acredito que é necessário cobrar algumas } \\
\text { atividades de outras contas quando os fundos de } \\
\text { orçamento para essas atividades já se esgotaram. } \\
\text { Eu tenho que alterar figuras relacionadas a operações } \\
\text { que reduzam variâncias de orçamento. } \\
\text { Eu acho que é necessário interromper algumas } \\
\text { atividades no meu departamento quando os fundos de } \\
\text { orçamento estão esgotados. }\end{array}$ & $\begin{array}{l}0,91 \\
0,76 \\
0,41\end{array}$ & 6,3 \\
\hline 5 & $\begin{array}{l}\text { INTERAÇÕES COM SUPERIORES } \\
\text { Eu trabalho com meu superior na preparação do } \\
\text { orçamento para o meu departamento. } \\
\text { Eu sou consultado pelo meu superior sobre os fatores } \\
\text { especiais que eu gostaria de ter incluído no orçamento } \\
\text { que está sendo preparado. } \\
\text { Eu vou até o meu superior para avisá-lo sobre como } \\
\text { alcançar meu orçamento. }\end{array}$ & $\begin{array}{l}0,51 \\
0,50\end{array}$ & 5,5 \\
\hline 6 & $\begin{array}{l}\text { ENVOLVIMENTO PESSOAL COM O ORÇAMENTO } \\
\text { Eu investigo as variâncias favoráveis e desfavoráveis } \\
\text { para o meu departamento. } \\
\text { A preparação do orçamento para o meu departamento } \\
\text { exige minha ajuda para um grande número de } \\
\text { detalhes. } \\
\text { Eu procuro pessoalmente variâncias de orçamento no } \\
\text { meu departamento. }\end{array}$ & $\begin{array}{l}0,52 \\
0,47\end{array}$ & 4,7 \\
\hline
\end{tabular}

As pontuações dos fatores para esses seis pontos foram usadas como as primeiras variações de comportamento orçamentário e podem ser descritas da seguinte forma: 
1. Explicações de variações necessárias: Alcance dos pedidos de explicação das variações e relato das ações tomadas para corrigir as causas das variâncias.

2. Influência nos planos de orçamento: Alcance do impacto nos planos do orçamento final.

3. Interações com os subordinados: Alcance das interações interpessoais com subordinados na preparação do orçamento e na análise das variações.

4. Reações ao endividamento previsto pelo orçamento: Frequiência de mudança de fundos de uma conta ou interrupção das atividades em frente de um orçamento "estourado".

5. Interações com os superiores: Alcance das interações interpessoais com superiores na preparação do orçamento ou na decisão de como alcançá-lo.

6. Envolvimento pessoal no orçamento: Alcance do envolvimento pessoal em atividades detalhadas do orçamento.

Além disso, pediu-se aos gerentes que estimassem a porcentagem de seu gasto de tempo em atividades relacionadas ao orçamento.

\section{Motivação e atitude}

A extensão com que o orçamento afetou a motivação intrínseca dos gerentes foi medida com a escala de três itens de Hackman e Porter (1968), posteriormente usados por Dermer (1975). Os gerentes indicaram seu consentimento, numa escala de 1 (discordo totalmente) a 5 (concordo totalmente), com afirmações que relacionam a realização das metas orçamentárias aos sentimentos de satisfação pessoal, realização, crescimento e desenvolvimento. Um exemplo de item é: "Bom desempenho relativo ao orçamento me dá um sentimento de realização". O alfa de Cronbach para essa escala foi altamente aceitável, 0,74.

As atitudes dos gerentes em relação ao orçamento foram medidas pelo uso de uma versão suavemente modificada do fator atitudinal descoberto nos estudos de Swieringa e Moncur (1975) e Bruns e Waterhouse (1975). Essa escala de cinco itens, sentimento de utilidade do orçamento, pede aos gerentes que julguem numa escala de cinco pontos se eles acreditam que o orçamento os permite (1) ser melhores gestores, (2) ser mais flexíveis, (3) ser mais inovadores, (4) ficar atentos a seus sucessos como gestores e (5) melhorar seus métodos de produção. 0 alfa Cronbach para essa escala foi 0,76.

\section{Desempenho}

Uma autoclassificação do desenvolvimento geral foi colhida pedindo aos gerentes para classificar seus departamentos numa escala de 1 (bom abaixo da média) a 5 (bom acima da média). Foi necessária uma medida de desempenho subjetiva porque é praticamente impossível alcançar apropriadamente os dados num estudo organizacional transversal. Autoclassificações foram usadas não apenas porque são relativamente fácies de ser obtidas, mas também porque evidências recentes (Heneman, 1974) sugerem que elas podem ser mais precisas e acuradas do que classificações feitas por superiores (como em estudos de pesquisa). Heneman sugere que é crível porque os superiores são tipicamente menos bem informados e mais sujeitos a erros de prestígio. 


\section{RESULTADOS}

\section{Correlações Interclasses}

Os tamanhos das 19 empresas variaram de 400 a 95.000 empregados. Os produtos oferecidos iam de componentes de semicondutores a equipamentos eletrônicos de larga escala, tais como computadores e sistemas de comunicação por microondas. Individualmente, no entanto, as companhias não eram bem diversificadas. Apenas seis das 19 compunham menos de $95 \%$ de suas receitas de negócios não-eletrônicos. Como esperado, o tamanho da corporação, a diversidade e a descentralização foram positivamente correlacionados uns com os outros, mas não significativamente no sentido estatístico.

Para facilitar a análise e a apresentação dos resultados, as pontuações dos fatores e outras variáveis de orçamento foram organizadas para corresponder às características hipotéticas do orçamento. Esse esquema de classificação está resumido na Tabela 2.

Tabela 2 -Relação entre as medidas de orçamento e as variáveis hipotéticas:

\begin{tabular}{|l|l|}
\multicolumn{1}{|c|}{$\begin{array}{c}\text { Característica } \\
\text { hipotética }\end{array}$} & \multicolumn{1}{c|}{ Variáveis } \\
\hline Participação & $\begin{array}{l}\text { Influência nos planos de orçamento } \\
\text { Envolvimento pessoal no orçamento } \\
\text { Tempo despendido com orçamento }\end{array}$ \\
\hline $\begin{array}{l}\text { Importância de se } \\
\text { alcançar o orçamento }\end{array}$ & $\begin{array}{l}\text { Exigência de explicações de variância } \\
\text { Reações ao endividamento previsto pelo orçamento. } \\
\text { Ligação com o sistema de remuneração da corporação. }\end{array}$ \\
\hline $\begin{array}{l}\text { Formalidade nas } \\
\text { comunicações }\end{array}$ & $\begin{array}{l}\text { Interaçães não-freqüentes com subordinados Interações não-freqüentes } \\
\text { com superiores } \\
\text { Detalhamento de informação }\end{array}$ \\
\hline $\begin{array}{l}\text { Sofisticação } \\
\text { sistema }\end{array}$ & $\begin{array}{l}\text { Sofisticação do apoio de computadores } \\
\text { Uso do manual de planejamento } \\
\text { Uso de orçamento de base-zero }\end{array}$ \\
\hline
\end{tabular}

A Tabela 3 apresenta a ordem de correlações entre as variáveis do orçamento. Uma vez que as variáveis foram codificadas, já que um aumento nas pontuações indica um uso mais administrativo do orçamento, a expectativa era de que as correlações positivas dominariam, e esse resultado é respaldado pelos dados. A maior exceção foi a variável do detalhamento da informação. Suas correlações com as outras variáveis são predominantemente negativas, indicando que um uso administrativo do orçamento é associado com menos detalhes de informação, ou maiores níveis de agregação. Além disso, a sofisticação do apoio computacional não parece ser uma parte importante de um uso administrativo do orçamento.

As variáveis dos resultados, a motivação e as atitudes gerenciais e o desempenho organizacional foram distribuídos conforme o esperado. Na média, os gerentes tiveram atitudes levemente favoráveis em relação ao alcance das metas orçamentárias e foram motivados por elas. A motivação e as atitudes intrínsecas foram altamente relacionadas $\left(r_{s}=0,74 p<0,10\right)$, mas as correlações entrem elas e o desempenho foram, virtualmente, zero. 
Tabela 3 - Classificação das correlações de Spearman entre as variáveis do orçamento $(\mathrm{N}=19)$ :

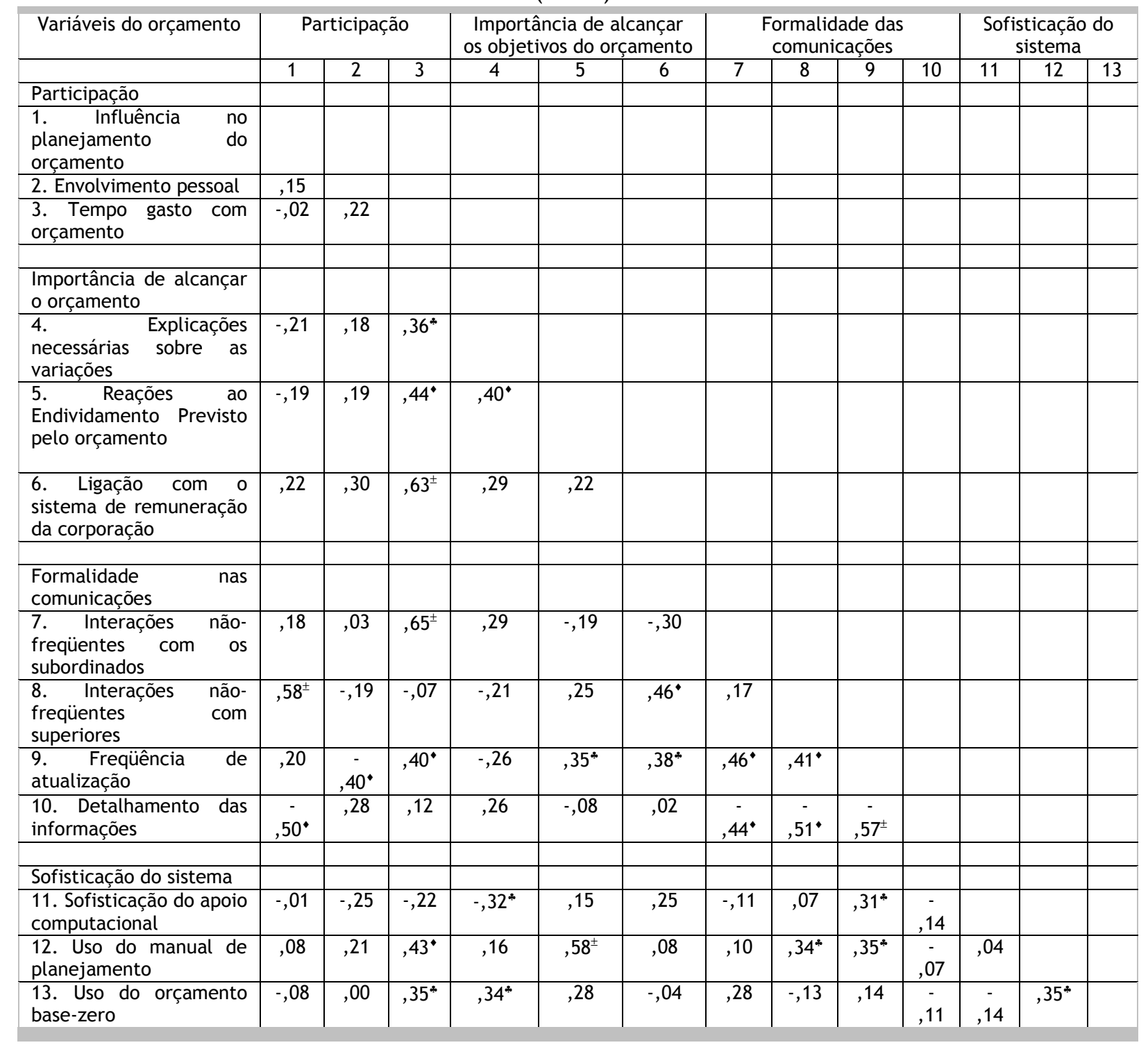

\section{Características corporativas e Orçamento (Hipótese I)}

As ordens das correlações entre as variáveis da corporação e do orçamento estão apresentadas na Tabela 4, dando respaldo a todas as partes da hipótese I. Gerentes nas empresas maiores, mais diversificadas e descentralizadas parecem participar mais no orçamento de maneira formal, com menores interações interpessoais diretas com seus subordinados e seus superiores, e sentem que alcançar as metas orçamentárias é importante para suas carreiras. O detalhamento da informação às variáveis da sofisticação dos computadores são, novamente, as maiores exceções às expectativas originais. 0

\footnotetext{
$* p<, 10$

$\mathrm{p}<, 05$

${ }^{ \pm} p<, 01$
} 
detalhamento parece ser maior nas corporações menores e a sofisticação dos computadores parece não estar relacionada com as variáveis corporativas.

Tabela 4 - Classificação das correlações de Spearman entre variáveis corporativas e orçamentárias $(\mathrm{N}=19)$

\begin{tabular}{|c|c|c|c|}
\hline \multirow{2}{*}{ Variáveis Orçamentárias } & \multicolumn{3}{|c|}{ Variáveis corporativas } \\
\hline & Tamanho & Diversificação & Descentralização $^{\mathrm{a}}$ \\
\hline \multicolumn{4}{|l|}{ Participação } \\
\hline 1. Influência no planejamento do orçamento & ,05 & 04 & $62^{* * *}$ \\
\hline 2. Envolvimento pessoal & ,14 & ,05 & ,35 \\
\hline 3. Tempo gasto com orçamento & $83^{* \pi+}$ & ,24 & ,24 \\
\hline \multicolumn{4}{|l|}{ Importância de alcançar o orçamento } \\
\hline 4. Explicações necessárias sobre as variaç̃os &, $38^{*}$ & $42^{* *}$ & ,04 \\
\hline 5. Reações ao endividamento previsto pelo orçamento &, $54^{* * *}$ & ,20 & ,10 \\
\hline $\begin{array}{l}\text { 6. Ligação com o sistema de remuneração da } \\
\text { corporação }\end{array}$ & $67^{* * *}$ & 12 &, $71^{* * *}$ \\
\hline \multicolumn{4}{|l|}{ Formalidade nas comunicações } \\
\hline 7. Interações não-freqüentes com os subordinados &, $50 * * *$ &, 30 &,- 35 \\
\hline 8. Interações não-freqüentes com superiores & $33^{*}$ & 07 &, 02 \\
\hline 9. Freqüêência de atualização & $40^{* *}$ &,- 18 & 18 \\
\hline 10. Detalhamento das informações &,- 23 &,- 05 &,- 08 \\
\hline \multicolumn{4}{|l|}{ Sofisticação do sistema } \\
\hline 11. Sofisticação do apoio computacional & 04 &,- 26 & 17 \\
\hline 12. Uso do manual de planejamento &, $70^{* * *}$ & 06 & 07 \\
\hline 13. Uso do orçamento base-zero & 29 &,- 18 & 00 \\
\hline
\end{tabular}

\section{Orçamento e motivações e atitudes (Hipótese 2)}

A Hipótese 2, que relaciona usos administrativos do orçamento com motivação e atitudes positivas, é apenas parcialmente observada. Os resultados apresentados na Tabela 5 mostram uma forte correlação positiva entre a participação e a importância de se alcançar as variáveis do orçamento e ambas a motivação e as atitudes. Contudo, nenhum padrão de correlação semelhante parece ligar a formalidade das comunicações e a sofisticação dos sistemas com a motivação e as atitudes dos gerentes. Esse resultado indica que esses efeitos cognitivos do orçamento são relacionados apenas às características específicas dos sistemas orçamentários. A ênfase que a organização dá ao orçamento e o alcance da participação permitida parecem ser particularmente favoráveis. Não houve evidência de que essas relações variaram interativamente com quaisquer variáveis medidas nesse contexto.

\footnotetext{
${ }_{\mathrm{a}}^{\mathrm{N}} \mathrm{N}$ 14; os dados necessários não puderam ser obtidos em 5 empresas.

$p<, 05$

${ }^{*} \mathrm{p}<, 10$

$p<, 05$
} 
Tabela 5

Classificação das correlações de Spearman entre orçamento e variáveis de motivação/atitude $(\mathrm{N}=19)$ :

\begin{tabular}{|c|c|c|}
\hline \multirow[b]{2}{*}{ Variáveis Orçamentárias } & \multicolumn{2}{|c|}{ Variáveis de motivação e de atitude } \\
\hline & $\begin{array}{l}\text { Motivação } \\
\text { Intrínseca }\end{array}$ & $\begin{array}{l}\text { Sentimento de utilidade } \\
\text { do orçamento }\end{array}$ \\
\hline \multicolumn{3}{|l|}{ Participação } \\
\hline 1. Influência no planejamento do orçamento & ,19 & ,18 \\
\hline 2. Envolvimento pessoal & ,41 &, 33 \\
\hline 3. Tempo gasto com orçamento &, 50 &, $47^{-1 *}$ \\
\hline \multicolumn{3}{|l|}{ Importância de alcançar o orçamento } \\
\hline 4. Explicações necessárias sobre as variações &, $45^{*}$ & , 39* \\
\hline 5. Reações ao Endividamento Previsto pelo orçamento & ,24 & ,28 \\
\hline 6. Ligação com o sistema de remuneração da corporação & ,70" & ,68" \\
\hline \multicolumn{3}{|l|}{ Formalidade nas comunicações } \\
\hline 7. Interações não-freqüentes com os subordinados & ,59" &, $61^{m+1}$ \\
\hline 8. Interações não-freqüentes com superiores &,- 12 &,- 11 \\
\hline 9. Freqüếncia de atualização & ,05 & ,09 \\
\hline 10. Detalhamento das informações &, 10 &,- 11 \\
\hline \multicolumn{3}{|l|}{ Sofisticação do sistema } \\
\hline 11. Sofisticação do apoio computacional &,- 10 & ,04 \\
\hline 12. Uso do manual de planejamento & ,18 & ,07 \\
\hline 13. Uso do orçamento base-zero & ,09 &,- 07 \\
\hline
\end{tabular}

\section{Orçamento e Desempenho organizacional (Hipótese 3)}

Para testar o contexto e o efeito interativo da estratégia de controle no desempenho, a população foi dividida na média do tamanho da corporação $(8.000$ empregados). A variável do tamanho foi escolhida para testar a interação por conta das limitações de dados das outras variáveis do contexto. A amostra não foi bem distribuída quanto à diversificação, já que 13 das 19 empresas produziam ao menos $95 \%$ de suas receitas de negócios eletrônicos, e as medidas centrais estavam ausentes para cinco empresas.

Como se afirma na Hipótese 3, esperava-se que os indicadores do uso administrativo do orçamento fossem mais favoravelmente relacionados à autoclassificação do desempenho nas empresas maiores e que os indicadores do uso interpessoal fossem mais favoravelmente relacionados às classificações de desempenho nas empresas menores. Os resultados apresentados na Tabela 6 fornecem algumas evidências da existência do efeito interativo do tamanho e uso orçamentários nos níveis de desempenho. Sete dos coeficientes do ranking de correlação entre usos administrativos do orçamento e os níveis de desempenho estão mostrados em itálico, porque eles são, ao menos, 0,3 mais altos para as empresas maiores do que para as empresas menores e nenhuma das diferenças é maior que 0,30 na direção oposta. Além disso, um teste de sinais (Siegel, 1956) foi feito com as diferenças e a probabilidade de 9 das 11 diferenças ser positiva é de 0,03. Esses resultados fornecem provas para a noção teórica de contingência geral, pois os usos administrativos do orçamento, especialmente a alta participação e maior importância colocada ao alcance das metas orçamentárias, parecem ser mais fortemente relacionados com um bom desempenho nas empresas maiores do que nas menores. 
A Tabela 6 fornece algumas evidências de ligação entre aspectos do orçamento e autoclassificação do desempenho que podem ser generalizadas para toda a amostra. A aparente diminuição da autonomia causada pela freqüência de reuniões com os superiores e pela falta de influência no planejamento orçamentário podem afetar desfavoravelmente o desempenho, embora a ligação causal possa estar em outra direção, com os superiores tornando-se mais envolvidos nas atividades de departamentos retardatários. OE orçamento de base-zero parece ter efeito negativo.

Tabela 6 - Classificação das correlações de Spearman entre variáveis orçamentárias e desempenho:

\begin{tabular}{|c|c|c|c|}
\hline \multirow[b]{2}{*}{ Variáveis Orçamentárias } & \multicolumn{3}{|c|}{ Variáveis de motivação e de atitude } \\
\hline & $\begin{array}{l}\text { Amostra total } \\
\quad(\mathrm{N}=19)\end{array}$ & $\begin{array}{c}\text { Empresas } \\
\text { menores } \\
(\mathrm{N}=9)\end{array}$ & $\begin{array}{l}\text { Empresas maiores } \\
\qquad(\mathrm{N}=10)\end{array}$ \\
\hline \multicolumn{4}{|l|}{ Participação } \\
\hline $\begin{array}{l}\text { I. Influência no planejamento do } \\
\text { orçamento }\end{array}$ & $48^{n+m}$ & ,27 &, 36 \\
\hline 2. Envolvimento pessoal &,- 04 &,- 40 & ,13 \\
\hline 3. Tempo gasto com orçamento & ,19 &,- 06 &, 24 \\
\hline \multicolumn{4}{|l|}{ Importância de alcançar o orçamento } \\
\hline $\begin{array}{l}\text { 4. Explicações necessárias sobre as } \\
\text { variações }\end{array}$ &,- 11 &,- 29 & ,13 \\
\hline $\begin{array}{l}\text { 5. Reações ao Endividamento Previsto } \\
\text { pelo orçamento }\end{array}$ & ,10 &,- 19 &, 30 \\
\hline $\begin{array}{l}\text { 6. Ligação com o sistema de remuneração } \\
\text { da corporação }\end{array}$ & 07 &,- 14 & 22 \\
\hline \multicolumn{4}{|l|}{ Formalidade nas comunicações } \\
\hline $\begin{array}{l}\text { 7. Interações não-freqüentes com os } \\
\text { subordinados }\end{array}$ & ,02 &,$- 47^{*}$ & ,24 \\
\hline $\begin{array}{l}\text { 8. Interações } \\
\text { superiores }\end{array}$ & $70^{* * \pi}$ & $76^{* * \pi}$ & ,71" \\
\hline 9. Freqüência de atualização & ,23 & ,31 &, 06 \\
\hline 10. Detalhamento das informações &,- 19 &,- 44 & ,04 \\
\hline \multicolumn{4}{|l|}{ Sofisticação do sistema } \\
\hline 11. Sofisticação do apoio computacional & 18 & ,11 & ,39 \\
\hline 12. Uso do manual de planejamento & 27 & ,42 & $\mathrm{a}$ \\
\hline 13. Uso do orçamento base-zero & Kenneth ,A3 M̈erchant & $\mathrm{b}$ &,$- 78^{n+m}$ \\
\hline
\end{tabular}

\section{DISCUSSÃO}

Este estudo de nível corporativo foi delineado para abordar o orçamento em nível sistêmico, em vez de focar uma ou mais características específicas, como a participação. Os sistemas orçamentários são multidimensionais e operam em conjuntos altamente variados, e para um escopo tão amplo, é necessário entender complexas redes de interdependência entre as variáveis. 0 objetivo deste estudo foi relacionar as

$$
\begin{aligned}
& { }^{* *} p<, 05 \\
& { }^{*} p<, 10 \\
& p<, 01
\end{aligned}
$$


características orçamentárias às descrições de variáveis do contexto corporativo e dos resultados individuais e organizacionais. 0 modelo para o estudo foi apresentado na Figura 1.

Evidências fornecidas por Bruns e Waterhouse (1975) e usadas aqui sugerem que muitas características do sistema orçamentário tendem a co-variar. Assim, pode ser possível explicar muitas variações do sistema orçamentário observado num número limitado de dimensões, como aquela aqui descrita, que abrange desde o uso administrativo até o uso interpessoal do orçamento.

As evidências fornecidas por Bruns e por Waterhouse num estudo multiindustrial e as deste estudo, considerando um único segmento industrial, sugerem que o modelo de orçamento varia com o contexto corporativo. Como se afirmou na Hipótese 1, empresas maiores, mais diversificadas e descentralizadas tendem a usar o orçamento de maneira administrativa, dando maior importância para o alcance das metas orçamentárias, maior participação dos gerentes de nível médio nas atividades relacionadas ao orçamento, padrões de comunicação mais formais e uso de suportes orçamentários mais sofisticados. Empresas menores e mais centralizadas tendem a contar mais com a supervisão direta dos gerentes de níveis mais altos e com freqüentes interações interpessoais e menos com comunicações mais formais.

Essas tendências ou arquétipos dos sistemas orçamentários parecem ser relacionadas às variáveis de resultados pessoais estudadas - motivação e atitudes dos gerentes - como antecipado na Hipótese 2, mas as relações são devidas às ligações significativas entre apenas algumas características específicas do orçamento e o resultado das variáveis. A motivação intrínseca e as atitudes em relação ao orçamento foi maior onde os gerentes participaram mais efetivamente em atividades relacionadas ao orçamento. Eles sentiram que o alcance das metas orçamentárias era mais importante e freqüentemente interagiram menos com seus subordinados.

Talvez o mais importante seja a evidência fornecida como suporte à noção teórica da contingência de um "casamento" desejável entre o contexto corporativo e os usos orçamentários, como colocado na Hipótese 3. As autoclassificações de desempenho foram maiores nas grandes empresas que usavam uma abordagem mais administrativa para o orçamento e nas empresas menores que o usavam com uma abordagem mais interpessoal.

Os resultados deveriam, no entanto, ser avaliados à luz das limitações do estudo. Primeiramente, a Figura 1 não apresenta um modelo totalmente geral. Ela poderia ser levada a outros níveis de análise, para incluir descritores de influências externas à organização, tais como a incerteza no ambiente de trabalho (Burns e Stalker, 1961; Gordon e Miller, 1976; Henderson e Nutt, 1978). Por outro lado, também poderia ser expandida para incluir descritores de partes da corporação, como as funções organizacionais (Hayes, 1977) e os tipos de indivíduo (Swieringa e Moncur, 1975).

Em segundo lugar, os dados podem ser enviesados pela amostra. É encorajador que os resultados do estudo, centrados num participante organizacional (gerentes de produção) em um tipo de indústria (eletrônica) apóiem aqueles obtidos por Bruns e Waterhouse (1975) que pesquisaram uma amostra diversa de gerentes num amplo escopo de indústrias. Todavia, ambos os estudos foram limitados às empresas que permitiram acesso a seus gerentes, e pode haver algumas tendenciosidades nesse auto-processo de seleção. Da mesma forma, os gerentes pesquisados não foram selecionados por acaso e, embora se tenha pedido aos entrevistados das corporações que conseguissem uma amostra representativa, isso pode ou não ter acontecido.

Em terceiro lugar, pode haver falta de algumas pequenas respostas enviesadas. Enquanto $85 \%$ das respostas gerais são extremamente boas para o levantamento da pesquisa, para quatro das 19 empresas, apenas a metade dos gerentes respondeu. 


\section{CONSIDERAÇÕES FINAIS}

Este estudo buscou analisar uma área muito complexa num nível de análise muito amplo. São necessários mais estudos desse tipo para ter seus resultados aplicados em estudos menores (por exemplo, indivíduos na empresa) nesta perspectiva.

Mesmo tendo sido grande a complexidade e as medidas disponíveis ainda estejam brutas, os resultados são encorajadores. Eles não apenas fornecem apoio aos resultados de estudos anteriores, mas sugerem que pode ser útil explicar as tendências orçamentárias em termos de variáveis mais agregadas, um agrupamento de variáveis multidimensionais que podem ser chamadas arquétipos ou gestalts. Dessa forma, quando a complexidade organizacional, por exemplo, é uma variável independente em estudo, em vez de enfocar uma característica orçamentária específica, como a participação ou a freqüência de feedback, os modelos orçamentários podem ser descritos de maneira mais útil e concisa, ficando entre os arquétipos chamados "administrativos" e "interpessoais". Ou, quando a incerteza no ambiente de trabalho é a variável independente em foco, os arquétipos podem ser rotulados de "mecanistisco" e "orgânico" (Burns e Stalker, 1961).

Além da necessidade de replicação desse estudo com melhores definições de arquétipos orçamentários e melhores instrumentos de medição, ampliações em, ao menos, duas direções são desejáveis: (1) estudos mais intensivos para examinar interações de variáveis tomadas uma de cada vez, como em campo experimental; e (2) estudos mais amplos, guiados pelos modelos de contingência, para que os dados possam ser obtidos de amostras escolhidas a fim de exaltar a variação das dimensões de interesse, enquanto controla-se os diversos fatores de interação possíveis que podem obscurecer ou distorcer os resultados. A complexidade é uma barreira significativa para o entendimento e só haverá progresso através de uma análise sistemática de uma quantidade considerável de dados em diferentes cenários, uma proposta árdua. Os benefícios, no entanto, são, provavelmente, significativos em termos de melhoria organizacional e desempenho individual, bem estar dos empregados e economia de custos.

\section{REFERÊNCIAS}

Ansari, S. (1977), "An Integrated Approach to Control System Design," Accounting, Organizations and Society (1977), pp. 101- 112.

(1979), "Towards an Open Systems Approach to Budgeting," Accounting, Organizations and Society (1979), pp. 149 - 161.

Banbury, J., and J. E. Nahapiet (1979), "Towards a Framework for the Study of the Antecedents and Consequences of Information Systems in Organizations" Accounting, Organizations and Society (1979), pp. 163 - 177.

Blau, P. M. and W. R. Scott (1962), Formal Organizations: A Comparative Approach (Chandler, 1962).

Brownell, P. (1981) "Participation in Budgeting, Locus of Control, and Organizational Effectiveness," The Accounting Review (October 1981), pp. 844 - 860.

Bruns, W. J., Jr. and J. H. Waterhouse (1975), "Budgetary Control and Organization Structure," Journal of Accounting Research (Autumn 1975), pp. 177- 203.

Burns, T., and G. M. Stalker (1961), The Management of Innovation (Tavistock, 1961)

Camman, C. (1976), "Effects of the Use of Control Systems," Accounting, Organizations and Society (1976), pp.301 - 313. 
Child, J. (1973), "Strategies of Control and Organizational Behavior," Administrative Science Quaterly (March 1973), pp. 1-17.

(1977), Organizations: A Guide to Problems and Practice (Harper and Row, 1977).

DeCoster, D. T., and J. P. Fertakis (1968); "Budget-Induced Pressure and Its Relationship to Supervisory Behavior," Journal of Accounting Research (Autumn 1968), pp. 237 - 246.

Dermer, J. D. (1975), "The Interrelationship of Intrinsic and Extrinsic Motivation," Academy of Management Journal (March 1975), pp. 751 - 763.

Foran, M. F., and D. De Coster (1974), "An Experimental Study of the Effects of Participation, Authoritarianism, and Feedback on Cognitive Dissonance in a Standard Setting Situation," The Accounting Review (October 1974), pp. 751 - 763.

French, J. R. P., J. Israel, and D. As (1960), "An Experiment on Participation in a Norwegian Factory," Human Relations (February 1960), pp. 3 - 19.

Galbraith, J. R., (1977), Organization Design (Addison Wesley, 1977).

Gordon, L. A., D. F. Larcker, and F. D. Tuggle (1978), "Strategic Design Process And the Design of Accounting Information Systems: Conceptual Linkages," Accounting, Organizations and Society (1978), pp. 203 - 213.

Gordon, L. A., and D. Miller (1976), "A Contingency Framework for the Design of Accounting Information Systems," Accounting Organizations and Society (1976), pp. 59 69.

Hackman, J. R., and L. W. Porter (1968), "Expectancy Theory Predictions of Work Effectiveness," Organizational Behavior and Human Performance (November 1968), pp. 417 - 426.

Hayes, D. C. (1977), "The Contingency Theory of Managerial Accounting," The Accounting Review (January 1977), pp. 22 - 39.

Henderson, J. C., and P. C. Nutt (1978), "On the Design of Planning Information Systems," Academy of Management Review (October 1978), pp. 774 - 785.

Heneman, H. G. (1974), "Comparisons of Self- and Superior Ratings of Managerial Performance," Journal of Applied Psychology (October 1974), pp. 638 - 642.

Hofstede, G. H. (1967), The Game of Budget Control (Van Gorcum, 1967).

Hopwood, A. G. (1972), "An Empirical Study of the Role of Accounting Data in Performance Evaluation," Empirical Research in Accounting: Selected Studies, 1972, supplement to Journal of Accounting Research, pp. 156 - 182.

(1974), "Leadership Climate and the Use of Accounting Data in Performance Evaluation," The Accounting Review (July 1974), pp. 485 - 495.

(1976), Accounting and Human Behavior (Prentice-Hall, 1976)

(1978), "Towards an Organizational Perspective for the Study of Accounting and Information Systems," Accounting, Organizations and Society (1978), pp.3 -13.

Inkson, J. H. K., D. S. Pugh, and D. J. Hickson (1970), "Organization Context and Structure: An Abbreviated Replication," Administrative Science Quarterly (September 1970), pp. 318 - 329.

Ivancevich, J. (1976), "Effects of Goal Setting on Performance and Job Satisfaction," Journal of Applied Psychology (October 1976), pp. 605 - 612.

Kast, F. E. and J. E. Rosenzweig (1973), Contingency Views of Organization and Management (Science Research Associates, 1973).

Kenis, I. (1979), "Effects of Budgetary Goal Characteristics on Managerial Attitudes and Performance," The Accounting Review (October 1979), pp. 707 - 721.

Lawrence, P. R., and J. W. Lorsch (1967), Organization and Environment: Managing Differentiation and Integration (Division of Research, Graduate School of Business Administration, Harvard University, 1967).

Luthans, F., and T. Stewart (1977), "A General Contingency Theory of Management," Academy of Management Review (April 1977), pp. 181 - 195.

Mealinea, L. W., and D. Lee (1979), "An Alternative to Macro-Micro Contingency Theories: An Integrative Model," Academy of Management Review (July 1979), p.. 318 - 345. 
Milani, K. (1975), The Relationship of Participation in Budget-Setting to Industrial Supervisor Performance Attitudes: A Field Study, The Accounting Review (April 1975), pp. $274-284$.

Moberg, D. J., and J. L. Koch (1975), "A Critical Appraisal of Integrated Treatments of Contingency Findings," Academy of Management Journal (March 1975), pp. 109 - 124.

Nie, N. H., C. H. Hull, J. G. Jenkings, K. Steinbrenner and D. H. Bent (1975), Statistical Package for the Social Sciences (McGraw-Hill, 1975).

Nunnally, J. C. (1967), Psychometric Theory (McGraw-Hill, 1967).

Otley, D. T. (1978), "Budget Use and Managerial Performance," Journal of Accounting Research (Spring 1978), pp. 122 - 149.

Ouchi, W. G., (1977), "The Relationship Between Organizational Structure and Organizational Control," Administrative Science Quarterly (March 1977), pp. 95 - 114.

and M. A. Maguire (1975), “Organizational Control: Two Functions," Administrative Science Quarterly (December 1975), pp. 559 - 569.

Pugh, D. S., D. J. Hickson, C. R. Hinings, and C. Turner (1969), "The Context of Organization Structure," Administrative Science Quarterly (March 1969), pp. 91 - 114.

Rumelt, R. P. (1974), Strategy, Structure and Economic Performance (Division of Research Graduate School of Business Administration, Harvard University, 1974).

Sathe, V. (1975), "Contingency Theories of Organizational Structure," in J. L. Livingstone, ed. Managerial Accounting: The Behavioral Foundations (grid, 1975), pp. 51 - 63.

Seafross, D. G. (1976), "Some Behavioral Aspects of Budgeting for Control: An Empirical Study," Accounting, Organizations and Society (1976), pp. 375 - 385.

Shepard, J. M., and J. G. Hougland (1978), "Contingency Theory: 'Complex Man' or 'Complex Organization?’” Academy Management Review (July 1878), pp. 413 - 427.

Siegel, S. (1956), Nonparametric Statistics for Behavioral Sciences (McGraw-Hill, 1956)

Stedry, A. C., and E. Kay (1966), "The Effects of Goal Difficulty on Performance: A Field Experiment," Behavioral Science (November 1966), pp. 459 - 470.

Steers, R. M. (1976), "Factors Affecting Job Attitudes in a Goal-Setting Environment," Academy of Management Journal (March 1976), pp. 6 - 16.

Swieringa, R. J., and R. H. Moncour (1975) Some Effects of Participative Budgeting on Managerial Behavior (National Association of Accountants, 1975).

Vroom, V. H. (1964), Work and Motivation (Wiley, 1964).

Waterhouse, J. H., and P. Tressen (1978), "A Contingency Framework for Management Accounting Systems Research," Accounting, Organizations and Society (1978), pp. 65 - 76.

Woodward, J, ed (1970), Industrial Organization: Behavior and Control (Oxford University Press, 1970). 